\title{
MicroRNA-370 suppresses the retinal capillary endothelial cell growth by targeting KDR gene
}

\author{
Wang XH, Chen L \\ Department of Ophthalmology, The First Hospital of China Medical University, Shenyang, \\ Liaoning Province, China. daiangle146@189.cn
}

\begin{abstract}
BACKGROUND: Ocular neovascularization (NV) is the one of the major causes of blindness in many ocular diseases. Currently, the administration of anti-VEGF agents has been widely accepted for the clinical management of these devastating diseases. However, the short effective duration and the non-responsive rate due to the protein nature of the anti-VEGF antibodies warrants further investigations to explore alternative angiogenic suppressants. Evidence suggested that microRNA-370 could inhibit the formation of vessels. However, the exact mechanism has not yet been clarified.

AIM: To investigate the regulatory role of microRNA-370 in the growth and apoptosis of retinal capillary endothelial cells and association between microRNA-370 and kinase insert domain-containing receptor (KDR) gene. METHODS: The effects of miRNA-370 on cell cycle and apoptosis, as well as the expression of cycle- and apoptosis-related genes, were determined using MTT, TUNEL assay, qRT-PCR, and Western blot. The direct target of miRNA-370 was confirmed using 3' untranslated region (UTR) luciferase reporter assay.

RESULTS: The miRNA-370 induced growth inhibition and apoptosis of retinal capillary endothelial cell while the inhibition of miRNA-370 reversed these effects. miRNA-370 upregulated the expression of CyclinD1, p21, p27, FasL, and Bim. Furthermore, miR-370 directly reduced the expression of KDR by targeting its 3'untranslated region. CONCLUSION: MicroRNA-370 inhibits the expression of KDR gene resulting in retinal capillary endothelial cell growth inhibition and apoptosis, which could be of value in the treatment of retinal neovascularization (Tab. 1, Fig. 5, Ref. 27). Text in PDF www.elis.sk. KEY WORDS: angiogenesis, miRNA-370, KDR, apoptosis, cellular proliferation.
\end{abstract}

\section{Introduction}

Ocular neovascularization (NV) is the primary cause of blindness in a wide range of ocular diseases such as diabetic retinopathy (DR), age-related macular degeneration (AMD), retinopathy of prematurity (ROP), central and branch retina vein occlusion (CRVO and BRVO), trauma and various inflammatory ocular diseases $(1,2)$. Retinal and choroidal vascular diseases constitute the most common causes of moderate and severe vision loss in developed countries (3). Vascular endothelial growth factor (VEGF) plays a central role in the development of several types of ocular NV. Currently, the administration of anti-VEGF agents has been widely accepted for the clinical management of these devastating diseases (4-6). However, the short duration of effect and non-responsive rate due to the protein nature of the anti-VEGF antibodies warrant further investigations to explore alternative angiogenic suppressants $(7,8)$.

Evidence suggested that microRNA-370 could inhibit the formation of vessels by targeting at the gene expression. Advance-

Department of Ophthalmology, The First Hospital of China Medical University, Shenyang, Liaoning Province, China

Address for correspondence: L. Chen, Department of Ophthalmology, The First Hospital of China Medical University, No.155, Nanjing Bei Street, Heping District, Shenyang, Liaoning Province, 110001, China. Phone: +8602483282630 ments in understanding the basic mechanisms of the angiogenic process have led to the identification of several macromolecules containing the potential capacity of angiostasis (1). Among the reported data, microRNAs, endogenous non-coding RNA molecules, demonstrated a promising capacity in regulating the cell proliferation, migration and transdifferentiation by regulating the gene translation post-transcriptionally through the pairing with the 3 '-untranslated region of the target DNA $(9,10)$.

Inhibition of VEGF signaling has shown to be greatly promising for the treatment of ocular neovascular disease. KDR is one of the promising therapeutic targets to be manipulated in order to stop the angiogenic process, which is a complex process involving many cells and cytokines. Among the large number of growth factors involving in the angiogenesis, VEGF plays a predominant role in the process by signaling through its ligand on the cellular membrane. The binding of VEGF to its receptor initiates a signal transduction cascade mediating vascular endothelial cell proliferation and migration, which eventually results in the formation of abnormal new vessels (11). VEGF receptor-2 (also known as KDR) is encoded by major membrane receptor for VEGF signaling (12). Previous report showed that the blocking of KDR with a specific antibody could result in the prevention of new vessel formation (13). The inhibition of KDR was reported to reduce the size of the choroidal neovascularization in animal models (14). Furthermore, it has been suggested that the microRNA-370 
might be able to regulate the expression of VEGF receptor-2 by binding to KDR gene, which could decrease the sensitivity of the cells to the increased level of VEGF. Therefore, we constructed the microRNA-370 overexpression and inhibition model of retinal capillary endothelial cells to examine the regulatory role of microRNA-370 in cellular proliferation, apoptosis and angiogenesis.

\section{Material and methods}

\section{Cell culture}

Human retinal capillary endothelial cells (HRCEC, JENNIO biological technology, Guangzhou, PRC) were maintained in HRCEC medium provided by the manufacturer incubated at 37 ${ }^{\circ} \mathrm{C}$ with $5 \% \mathrm{CO}_{2}$.

\section{Lentivirus construction and transfection}

Establishment of cell lines expressing miR-370

Lentiviral vectors were used to establish stable cell lines expressing miR-370. Psin-EF2-miR-150-IRES-GFP-puro expressing miR-370 pre-miRNA was constructed using specific primers, 5'GCCGAATTCTTGAACTAATTCATTTCTTT - $3^{\prime}$ (forward) and 5'-GCCGGATCCCGTCTCTGTGCCTGTTTCCC -3' (reverse). The $293 \mathrm{~T}$ cells were used to generate lentivirus containing the sequences of miR-370 precursor. The 293T cells were co-transfected with pMD2.G, pSPAX2 and Psin-EF2-miR-370-IRESGFP-puro using Lipofectamine 2000 Transfection Regent (Invitrogen, Waltham, MA). After $48 \mathrm{~h}$, the supernatant was collected and filtered using 0.22 um filter. Filtered supernatant containing lentivirus was then used for transduction.

HRCEC cells were plated on 6-well plates and incubated to $40 \%$ confluence. The cells were infected with lentivirus for $4 \mathrm{~h}$ and cultured with MEM medium containing $10 \%$ of FBS and including polybrane $(4 \mu \mathrm{g} / \mathrm{mL})$. Then the medium was changed to MEM medium containing $10 \%$ of FBS and the infection procedure was repeated. Afterwards, the cells were incubated for 48 hours in fresh MEM medium containing $10 \%$ of FBS. Expand cells were then used for further experiments and transduction efficiency was measured by a fluorescence microscope (Olympus, Japan). A control cell line transducted with empty vectors was also established using the same procedure.

\section{Cell growth assay}

HRCEC cells $\left(1 \times 10^{4}\right)$ expressing miRNA-370 or inhibitors were cultured in a 24 -well plate for 4 days, and the cell number of the cells was determined every day. This assay was performed in triplicate and repeated in three independent experiments.

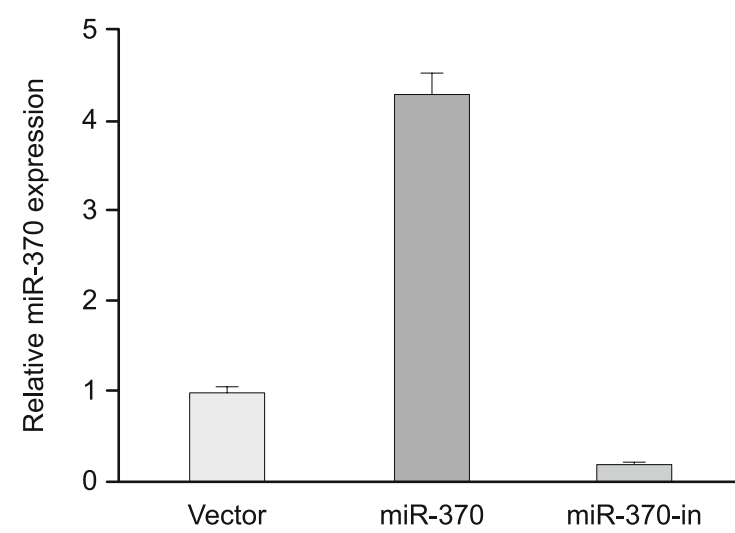

Fig. 1. The relative expression level of miRNA-370 in the human retinal capillary endothelial cells containing miRNA-370 vector (miR370), miRNA-370 inhibitor (miR-370-in) and blank vector (Vector).

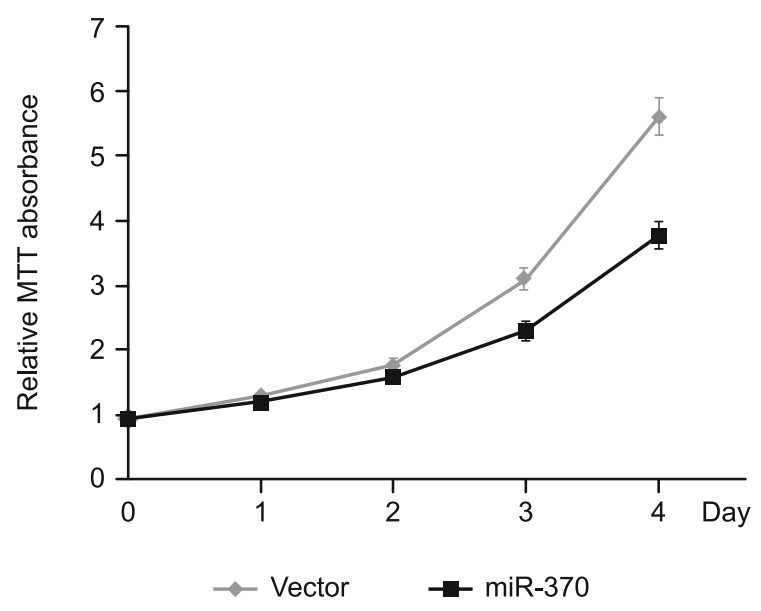

Fig. 2. The miRNA-370 inhibited the proliferation of HRCEC cells. The cells that overexpressed miRNA-370 (miR-370) showed a lower proliferation ratio at all time points compared to the cells treated with blank vectors (vector).

Terminal deoxynucleotidyl transferase dUTP nick end labeling (TUNEL) assay

TUNEL assay combined with propidium iodide (PI) staining were used to identify the ratio of apoptosis versus total cell number. Briefly, the HRCEC cells cultured in high glucose culture medium ( $33 \mathrm{mmol} / \mathrm{L})$ were transfected with microRNA-370 vector or inhibitor as aforementioned. Afterwards, the cells were fixated by $4 \%$ paraformaldehyde for 5 minutes. Then, the cells were stained

Tab. 1. The primer sets for Real-time PCR.

\begin{tabular}{lll}
\hline Primers & Forward & Reverse \\
\hline p21 & AGTCAGTTCCTTGTGGAGCC & CATGGGTTCTGACGGACAT \\
p27 & AAGAAGCCTGGCCTCAGAAG & TTCATCAAGCAGTGATGTATCTGA \\
Bim & CCTCCCTACAGACAGAGCCA & GATAGTGGTTGAAGGCCTGG \\
FasL & GCACACAGCATCATCTTTGG & GGACCTTGAGTTGGACTTGC \\
GAPDH & GACTCATGACCACAGTCCATGC & AGAGGCAGGGATGATGTTCTG \\
\hline
\end{tabular}



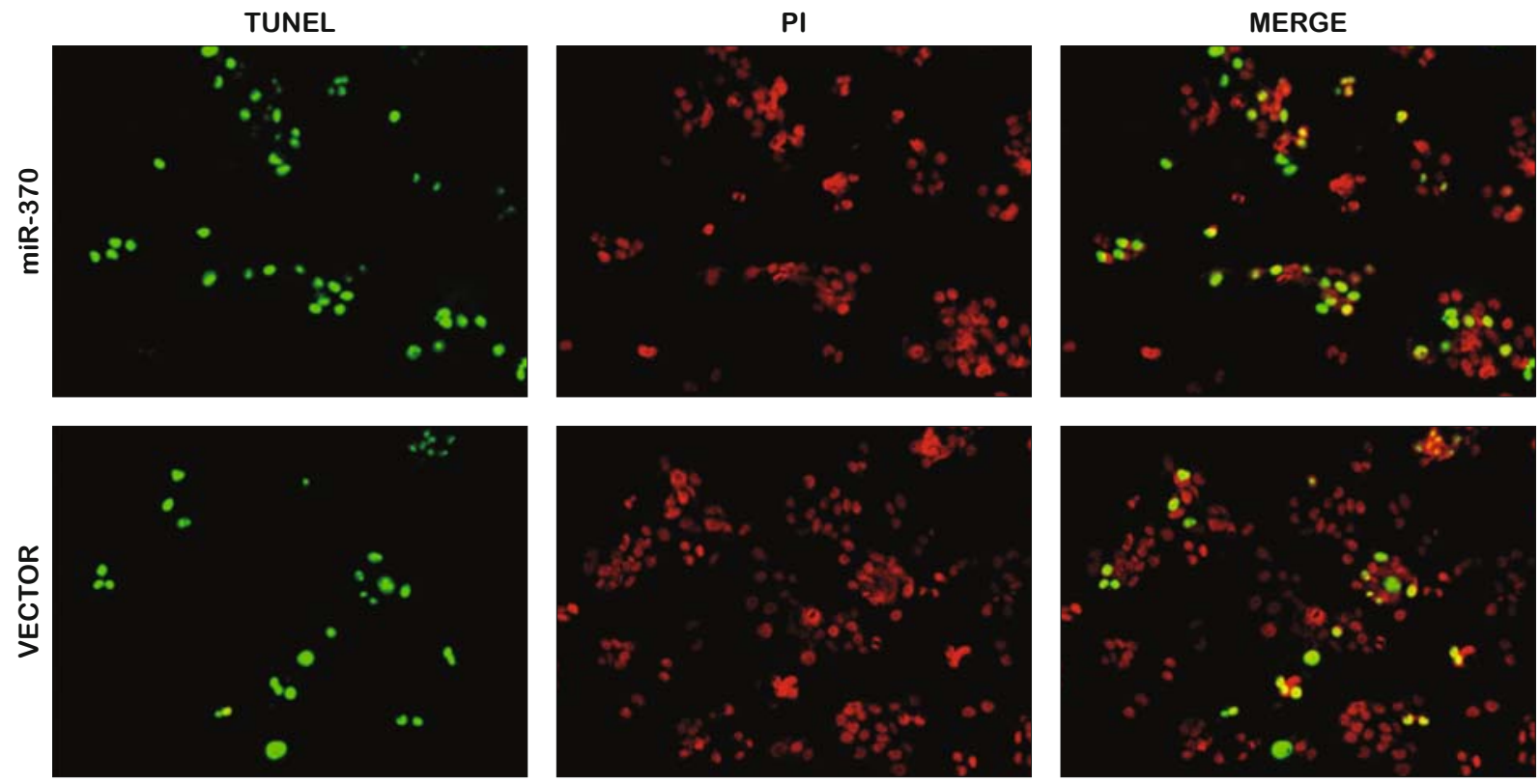

(A)
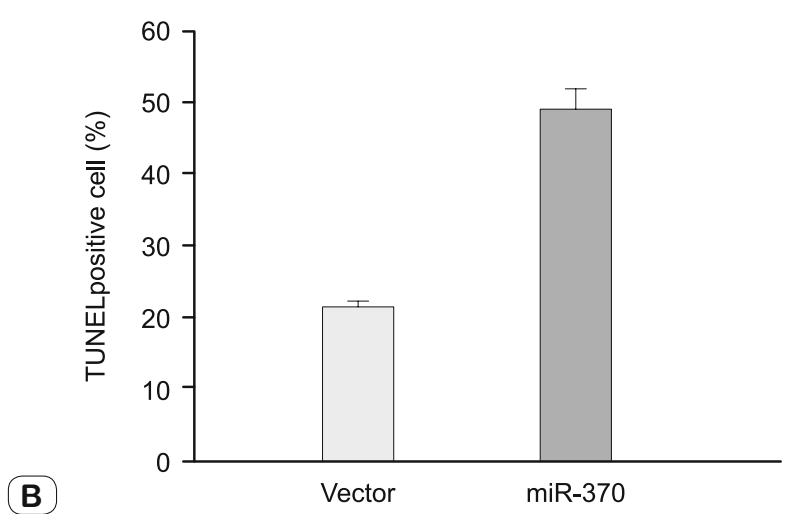

Fig. 3. The miRNA-370 induced apoptosis of human retinal capillary endothelial cells. A: The HRCEC cells were transfected with miRNA-370 vector or blank vector for $\mathbf{4 8}$ hours and the apoptosis was determined by TUNEL (green) assay and counter-stained with propidium iodide (PI, red). B: The percentage of TUNEL-positive cells was counted in the histogram, which showed a significantly higher positive rate in the miRNA-370 overexpressing cells.

with FITC-conjugated dUTP (green) and PI (red) follow the protocol of the manufacturer. The apoptotic cells and the total cells' number were counted using a fluorescent microscope (Olympus, Japan). The assays were triplicated.

\section{Western blot}

Whole-cell lysates were extracted from cells suspended in the immune precipitation buffer (Beyotime, China). Lysates were separated by electrophoresis on polyacrylamide gels containing $0.1 \%$ sodium dodecyl sulfate (SDS-PAGE) and then transferred to polyvinylidene difluoride membranes. The membranes were blocked with $5 \%$ non-fat milk in Tris Buffer Saline Tween 20 (TBST) buffer. The blots were incubated with the primary antibody and exposed to horseradish peroxidase (HRP)-conjugated secondary antibodies (goat anti mouse IgG or goat anti rabbit IgG depending on the primary antibody) afterwards. The bands on the membrane were visualized and captured using the ECL reagent (Beyotime) and documented on X-ray films. The pixel densities of proteins were quantified using ImageJ 1.47 software. Antibodies against to p27, p21, FasL, Bim and KDR as well as (HRP)-linked antimouse and -rabbit IgG, were bought from Beyotime.

\section{Real-time PCR}

Total RNA was isolated from HRCEC cells using trizol (Invitrogen, Carlsbad, CA) and reverse transcription of mRNA was performed using QuantScript RT kit (Tiangen) according to the manufacturer's protocol. RT-PCR was performed with SYBR Green Realtime PCR Kit (TOYOBO, JAPAN) and the Chromo4 ${ }^{\mathrm{TM}}$ Real-Time PCR System (Bio-bad, US). The specific primer sequences for the genes are shown in Table 1. Relative mRNA expression was carried out using $2-\Delta \Delta \mathrm{Ct}$ method after normalization to GAPDH. 

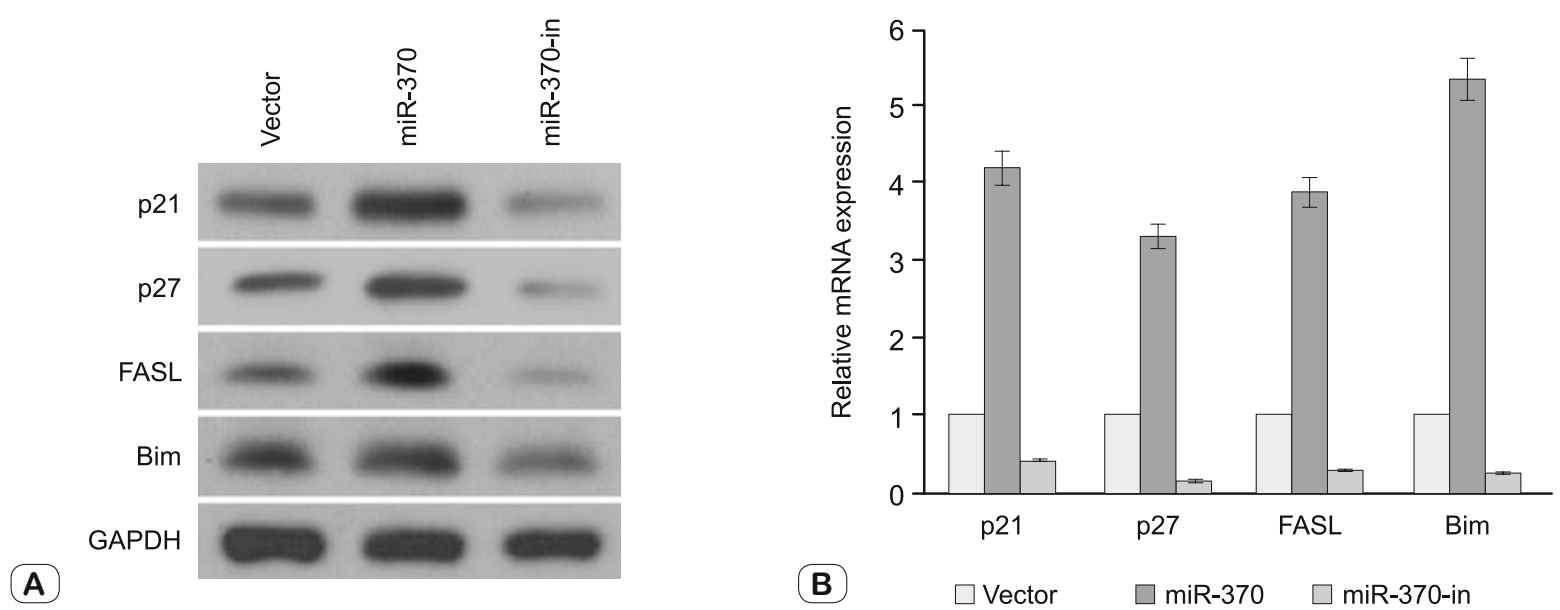

Fig. 4. The miRNA-370 inhibits the cellular proliferation and promotes apoptosis by upregulating the expression of p27, p21, FasL and Bim, which were demonstrated by the western blot (A) and quantitative PCR (B). The HRCEC cells overexpressing miRNA-370 (miR-370) produced significantly higher level of the aforementioned factor while the miRNA-370 inhibitor resulted in the downregulation of these factors.

\section{Luciferase reporter assay}

Wild type or mutant $3^{\prime}$ untranslated region (UTR) of KDR containing the putative binding sites for miRNA-370 were subcloned into psiCHECK-2 Vectors. The constructed plasmids were transfected into HRCEC cells as well as cells expressing miR-370 or inhibitors using Lipofectamine 2000 Transfection Regent (Invitrogen). After $48 \mathrm{~h}$, the luciferase activity in cell lysates was evaluated using the Dual-Luciferase Reporter Assay system according to the manufacturer's instructions (Promega, USA).

\section{Statistical analysis}

Statistical significance for the experiments was determined using the Student's $t$ test or One-way ANOVA. $p<0.05$ was regarded as statistically significant.

\section{Results}

miRNA-370 induced growth inhibition and apoptosis of HRCEC cells

To investigate the role of miRNA-370 in the retinal angiogenesis, the HRCEC cells were transfected with either the miRNA-370 vectors, miRNA-370 inhibitor or blank vector. The expression of miRNA-370 in each cell lines was measured by RT-PCR, which showed that the cells transfected with miRNA-370 vector had the highest level of miRNA-370 expression while the miRNA-370 inhibitor suppressed the expression of miRNA-370 (Fig. 1). The relative proliferation ratios of the miRNA-370 overexpressing cells and the blank vector cells were calculated by using the normal HRCEC cells as the house hold control using MTT assay. The results showed that the proliferation ratios were significantly higher in the cells treated with blank vectors compared to that in the cells overexpression miRNA-370, which suggested that miR-370 inhibited the proliferation of HRCEC cells (Fig. 2). Additionally, the Tunnel assay showed that the overexpression of miRNA-370 in the HRCEC cells promoted apoptosis of HRCEC cells (Fig. 3).
The miRNA-370 upregulated the expression of CyclinD1, p21, p27, FasL, and Bim in the HRCEC cells

The overexpression of miRNA-370 in the HRCEC cells upregulated the expression of CyclinD1, p21 and p27 which are known regulators inhibiting the cellular proliferation by regulating the cell cycle progression. Additionally, the results showed that the HRCEC cells producing high level of miRNA-370 expressed higher level of two pro-apoptotic genes, namely, FasL, and Bim, both on the protein level and mRNA level. The results were confirmed by both western-blot and quantitative PCR (Fig. 4). The inhibition of miRNA-370 resulted in the downregulation of the aforementioned factors.

The miR-370 directly reduced the expression of KDR by targeting its 3' untranslated region (3'UTR)

We found significant downregulation of KDR gene in the HRCEC cells overexpressing miRNA-370. The inhibition of miRNA-370 resulted in the upregulation of KDR (Fig. 5). To confirm the direct interaction between miRNA-370 and 3'UTR or KDR, two luciferase reporter plasmids containing either the wild type or

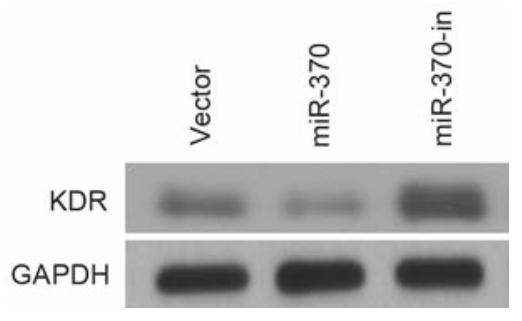

Fig. 5. miRNA-370 regulates the expression of KDR by the direct targeting at the 3'UTR of KDR.

The protein expression of KDR was downregulated in miRNA-370 overexpressing cells and upregulated in cells that the miRNA- 370 was inhibited. B: The sequence of the wild type and mutant 3'UTR of KDR 
mutant 3'UTR were constructed. In HRCEC cells overexpressing miRNA-370, the expression of KDR was significantly lower than the wild type HRCEC cells and HRCEC cells transfected with miRNA-370 inhibitors (Fig. 5).

\section{Discussion}

The results of the current study added further information about the correlation between increased miRNA-370 and ocular neovascularization. We observed anti-angiogenic effect of miRNA-370 as miRNA-370 inhibited the proliferation and promoted apoptosis of retinal capillary endothelial cells. Additionally, KDR gene was identified as a direct target of miRNA-370 which is directly linked to the formation of new vessels by regulating the expression of CyclinD1, p21, p27, FasL, and Bim. Therefore, we propose a hypothesis that miRNA-370 induces the transcriptional arrest of KDR by the binding of 3'-UTR and the upregulation of CyclinD1, p21, p27, FasL, and Bim, which eventually results in the suppression of capillary endothelial cell proliferation and the promotion of apoptosis.

Our results showed that miRNA-370 is pro-apoptotic which could inhibit the proliferation of HRCEC cells. The inhibitory function of miRNA-370 in the proliferation and pro-apoptotic effect has also been reported in other types of cells. It has been shown that miR-370 inhibited cell proliferation of liver cancer cells by PI3K/Akt signaling pathway (9). The regulatory role of miRNA-370 in the cellular proliferation were reported during embryogenic process as well as a few types of cancer cells $(10,15$, 16). The mature miRNA recognized the target mRNAs by incorporating into a RNA-induced silencing complex (RISC) resulting in translational inhibition or destabilization of the target mRNA. Our results showed that the overexpression of miRNA-370 resulted in upregulation of FasL, and Bim, which are the known pro-apoptotic factors $(17,18)$. Thus, the pro-apoptotic effect of miRNA-370 is functioned through these factors. Furthermore, the current study showed that the overexpression of miRNA-370 increased the expression of CyclinD1, p21 and p27, which are related to the regulation of cell cycle progression $(19,20)$.

The current study identified the KDR gene as a novel target of miRNA-370 as miR-370 suppressed the transcriptional process of KDR through targeting the 3'UTR of its mRNA. The direct targeting of miRNA-370 to the 3'UTR of the KDR gene shed some new light on the novel therapeutic approach to the ocular neovascularization. Although VEGF binds to VEGFR-1 with high affinity, and less strongly to VEGFR-2 (21). The tyrosine kinase (TK) activity of KDR is about 10 fold stronger than VEGFR-1. Along with the results of gene knockout studies, previous reports indicated that the major signal transducer in angiogenesis is VEGFR-2 (12). The inhibition of KDR activities has been considered to be one of the potential promising targets for the prevention of neovascularization. Ahani et al reported that the nanobody carried by lentiviral vectors targeting the KDR could potentially reduce the neovascularization in tumor vasculature (22). Other authors have also reported various approaches to inhibit the biological activity of KDR thus resulting in the inhibition of angiogenesis $(23,24)$.
Since the VEGR-KDR axis has been reported to be involved in the cell migration, proliferation, differentiation as well as transducing cell survival signals, it is possible that the upregulation of CyclinD1, p21, p27, FasL, and Bim in the HRCEC cells is mediated by VEGF-KDR axis as well (25-27).

\section{References}

1. Dorrell M, Uusitalo-Jarvinen H, Aguilar E, Friedlander M. Ocular neovascularization: basic mechanisms and therapeutic advances. Surv Ophthalmol 2007; 52 Suppl 1: S3-19.

2. Walz JM, Bemme S, Pielen A et al. The German ROP Registry: data from 90 infants treated for retinopathy of prematurity. Acta Ophthalmol 2016.

3. Kinnunen K, Yla-Herttuala S. Vascular endothelial growth factors in retinal and choroidal neovascular diseases. Ann Med 2012; 44 (1): 1-17.

4. Tah V, Orlans HO, Hyer J et al. Anti-VEGF Therapy and the Retina: An Update. J Ophthalmol 2015; 2015: 627674.

5. Solomon SD, Lindsley K, Vedula SS, Krzystolik MG, Hawkins BS. Anti-vascular endothelial growth factor for neovascular age-related macular degeneration. Cochrane Database Syst Rev 2014; (8): CD005139.

6. Toth LA, Stevenson M, Chakravarthy U. Anti-vascular endothelial growth factor therapy for neovascular age-related macular degeneration: Outcomes in Eyes with Poor Initial Vision. Retina 2015; 35 (10): 1957-1963.

7. Rofagha S, Bhisitkul RB, Boyer DS, Sadda SR, Zhang K, Group S-US. Seven-year outcomes in ranibizumab-treated patients in Anchor, Marina, and Horizon: a multicenter cohort study (SEVEN-UP). Ophthalmology 2013; 120 (11): 2292-2299.

8. Todorich B, Yiu G, Hahn P. Current and investigational pharmacotherapeutic approaches for modulating retinal angiogenesis. Expert Rev Clin Pharmacol 2014; 7 (3): 375-391.

9. Sun G, Hou YB, Jia HY, Bi XH, Yu L, Chen DJ. MiR-370 promotes cell death of liver cancer cells by Akt/FoxO3a signalling pathway. Eur Rev Med Pharmacol Sci 2016; 20 (10): 2011-2019.

10. Duan N, Hu X, Yang X, Cheng H, Zhang W. MicroRNA-370 directly targets FOXM1 to inhibit cell growth and metastasis in osteosarcoma cells. Int J Clin Exp Pathol 2015; 8 (9): 10250-10260.

11. Shibuya M. VEGF-VEGFR Signals in Health and Disease. Biomol Ther (Seoul) 2014; 22 (1): 1-9.

12. Shalaby F, Rossant J, Yamaguchi TP et al. Failure of blood-island formation and vasculogenesis in Flk-1-deficient mice. Nature 1995; 376 (6535): 62-66.

13. McLeod DS, Taomoto M, Cao J, Zhu Z, Witte L, Lutty GA. Localization of VEGF receptor-2 (KDR/Flk-1) and effects of blocking it in oxygen-induced retinopathy. Invest Ophthalmol Vis Sci 2002; 43 (2): 474-482.

14. Kinose F, Roscilli G, Lamartina $S$ et al. Inhibition of retinal and choroidal neovascularization by a novel KDR kinase inhibitor. Mol Vis 2005; 11: 366-373.

15. Chen T, Gao F, Feng S, Yang T, Chen M. MicroRNA-370 inhibits the progression of non-small cell lung cancer by downregulating oncogene TRAF4. Oncol Rep 2015; 34 (1): 461-468.

16. Yungang W, Xiaoyu L, Pang T, Wenming L, Pan X. miR-370 targeted FoxM1 functions as a tumor suppressor in laryngeal squamous cell carcinoma (LSCC). Biomed Pharmacother 2014; 68 (2): 149-154. 
17. Kraft $\mathbf{P}$, Schleicher R, Olbrich $\mathbf{M}$ et al. Platelet derived FasL contributes to apoptosis in stroke. Thromb Haemost 2016; 116 (5): 998-1000.

18. Carter MJ, Cox KL, Blakemore SJ et al. PI3Kdelta inhibition elicits anti-leukemic effects through Bim-dependent apoptosis. Leukemia 2016.

19. Wang X, Guo H, Liu W et al. Effects of siRNA-Mediated Knockdown of HDAC1 on the Biological Behavior of Esophageal Carcinoma Cell Lines. Med Sci Monit 2016; 22: 1291-1296.

20. Chen Y, Gu J, Li D, Li S. Time-course network analysis reveals TNFalpha can promote $\mathrm{G} 1 / \mathrm{S}$ transition of cell cycle in vascular endothelial cells. Bioinformatics 2012; 28 (1): 1-4.

21. Sawano A, Takahashi T, Yamaguchi S, Aonuma M, Shibuya M. Flt-1 but not KDR/Flk-1 tyrosine kinase is a receptor for placenta growth factor, which is related to vascular endothelial growth factor. Cell Growth Differ 1996; 7 (2): 213-221.

22. Ahani R, Roohvand F, Cohan RA et al. Sindbis Virus-Pseudotyped Lentiviral Vectors Carrying VEGFR2-Specific Nanobody for Potential Transductional Targeting of Tumor Vasculature. Mol Biotechnol 2016; 58 (11): 738-747.
23. Kim DG, Jin Y, Jin J et al. Anticancer activity of TTAC-0001, a fully human anti-vascular endothelial growth factor receptor 2 (VEGFR-2/KDR) monoclonal antibody, is associated with inhibition of tumor angiogenesis. MAbs 2015; 7 (6): 1195-1204.

24. Salerno S, Marini AM, Fornaciari G et al. Investigation of new 2-aryl substituted Benzothiopyrano (4,3-d)pyrimidines as kinase inhibitors targeting vascular endothelial growth factor receptor 2. Eur J Med Chem 2015; 103: 29-43.

25. Matsumoto T, Bohman S, Dixelius J et al. VEGF receptor-2 Y951 signaling and a role for the adapter molecule TSAd in tumor angiogenesis. EMBO J 2005; 24 (13): 2342-2353.

26. Dias S, Shmelkov SV, Lam G, Rafii S. VEGF (165) promotes survival of leukemic cells by Hsp90-mediated induction of Bcl-2 expression and apoptosis inhibition. Blood 2002; 99 (7): 2532-2540.

27. Sase H, Watabe T, Kawasaki K, Miyazono K, Miyazawa K. VEGFR2-PLCgamma1 axis is essential for endothelial specification of VEGFR2+ vascular progenitor cells. J Cell Sci 2009; 122 (Pt 18): 3303-3311.

Received December 21, 2016. Accepted January 10, 2017. 